\title{
Increased Total Serum Alkaline Phosphate Is Associated With Enhanced Bone Mineral Density in Dogs With Interval Exercise Training
}

Hae Sung Lee

Hanyang University

Hyun Ju Oh

Chungnam National University

Jong-Hee Kim ( $\square$ carachel07@hanyang.ac.kr)

Department of Physical Education, College of Performing Arts and Sport, Hanyang University, 222 Wangsimni-ro, Seongdong-gu, Seoul, Republic of Korea

\section{Research Article}

Keywords: Physical activity, muscle physiology, geriatric syndrome, immune function

Posted Date: January 19th, 2021

DOI: https://doi.org/10.21203/rs.3.rs-146618/v1

License: (c) (i) This work is licensed under a Creative Commons Attribution 4.0 International License. Read Full License 


\section{Abstract}

There are growing attention and interdisciplinary efforts for One Health to attain optimal health and wellbeing for humans and animals. Exercise has been suggested as a powerful intervention for health care and fitness management in humans; however, few studies have demonstrated the beneficial effect of exercise on dogs. The purpose of this study was to examine the effects of exercise training on heart rate $(\mathrm{HR})$, bone mineral density (BMD), muscle volume (MV), and hematological and serum biomarkers in dogs. Six healthy beagles completed the interval treadmill exercise protocol, developed based on the FITT principle, two times a week for 12 weeks. For a physiological parameter evaluation, the mean HR value was analyzed using a Polar $\mathrm{H} 10$ system and software program. Quantitative computed tomography was used to determine BMD in the femur and vertebrae and MV in the thigh before and after exercise training. To perform hematological and serum biochemical parameter assessment, blood samples were analyzed at zero and 12 weeks of exercise. We show that interval exercise results in a normal HR response and no adverse behavioral and physiological effects on dogs. Exercise improves BMD in the femur (541.6 \pm 16.7 vs. $610.2 \pm 27.8 \mathrm{HA}, p<0.01$ ) and increases serum total alkaline phosphatase (TALP; $68.6 \pm 9.2$ vs. 81.3 $\pm 17.2, p<0.01)$, aspartate aminotransferase $(23.5 \pm 1.0$ vs. $33.5 \pm 1.6, p<0.01)$, and creatine kinase $(114.8 \pm 5.3$ vs. $214.0 \pm 20.8, p<0.01)$ levels. There is a positive relationship between BMD and TALP (femur: $\mathrm{r}=0.760, p=0.004$; vertebrae: $\mathrm{r}=0.637 ; p=0.025$ ). Our findings suggest that long-term interval exercise training is beneficial to increase BMD in the femur, and an increased TALP level is a concomitant mechanism for enhancing BMD with exercise in dogs.

\section{Introduction}

Dogs, a common companion animal, have coexisted with humans since the Neolithic Age and are recognized as family members in many modern societies ${ }^{1}$. Studies have reported that over $70 \%$ of dog owners feel affection for their canine companions akin to raising a baby ${ }^{2}$. As the bonds between dogs and humans grow stronger, there is greater demand for better canine health care and fitness management ${ }^{1}$. Exercise has been recognized as an essential component of "One Health", an interdisciplinary effort to attain optimal health and well-being for humans and animals ${ }^{2,3}$. Many human studies have robustly established that exercise improves health and fitness ${ }^{4-7}$, but research exploring the beneficial effects of exercise in dogs is limited.

Studies have shown that physiological and biochemical parameters are fundamental to investigate the effects of exercise on health, fitness, and function ${ }^{4-7}$. Heart rate ${ }^{7}$ is a primary physiological indicator for diagnosing cardiac function and aerobic performance. Exercise-induced adaptation in HR (i.e., reduction in resting HR) is related to improved cardiovascular fitness ${ }^{6}$. HR response during competitive exercise and recovery can also be used to assess heart-rhythm disorders, such as arrhythmia, in dogs ${ }^{6}$. HR levels influence cardiac output and $\mathrm{VO}_{2 \text { max }}$ during exercise ${ }^{7}$. Hematological and serum biochemical analyses can reveal systemic and metabolic functions. Treadmill exercise studies showed significant improvements in rectal temperature, glucose and lactate concentrations, red blood cell counts, 
hematocrit, and HR in dogs ${ }^{8,9}$. Although each parameter has its benefits, comprehensive examinations are required for a thorough screening of a dog's health and fitness.

Bone mineral density (BMD) and muscle volume (MV) are valuable measurements to assess overall health status and bone-muscle interactions in post-exercise dogs. Some studies have found that low BMD and MV are associated with several disorders, including inflammatory diseases, tumors, osteogenesis imperfecta, degenerative arthritis, and endocrine diseases ${ }^{10,11}$. Furthermore, exercise (e.g., sprinting, jogging, weightlifting, swimming) can be a powerful intervention for the prevention and treatment of these conditions in humans ${ }^{12-14}$. In several other studies, participants of different ages and genders showed positive changes in BMD, MV, and bone turnover serum biomarkers (e.g., bone-specific alkaline phosphatase, deoxypyridinoline, and calcium) after exercise designed according to the Frequency, Intensity, Time/duration, Type, Volume, and Progression (FITT-VP) Principle ${ }^{15-19}$. To date, however, there has been little scientific examination of the effect of exercise on bone and muscle health in dogs. One reason for this lack of knowledge is the ethical issue of studying bone and muscle tissues, which requires invasive procedures.

Non-invasive quantitative computed tomography (QCT) is a three-dimensional non-projection technique to evaluate BMD and MV. In human studies, QCT has been used to investigate muscle-bone interactions ${ }^{20,21}$. In veterinary medicine, however, QCT is mainly used to diagnose skeletal changes in osteoporosis and other metabolic bone diseases ${ }^{22-24}$. To date, there are no studies using QCT to assess changes in BMD and MV after exercise in dogs, nor have there been studies that examined the relationship between biomarker levels and bone-muscle properties. We postulated that it would be possible to use QCT to assess subtle changes in BMD and MV after treadmill exercise training. In this study, we modified a 12-week interval exercise protocol that yielded positive changes in physiological parameters for humans and applied it to healthy $\operatorname{dogs}^{4}$ to explore the effects of exercise and associated mechanisms by identifying changes in $\mathrm{HR}, \mathrm{BMD}, \mathrm{MV}$, and bone serum markers.

\section{Results}

Heart rate. Figure 1A shows the HR response during interval exercise, which includes a series of workout (W) stages and incomplete resting (R) stages. HR was analyzed for all beagles who completed the interval exercise protocol. Mean HR during the $\mathrm{W}$ stage was significantly higher than that during the $\mathrm{R}$ stage $(p<0.01)$. To evaluate if the exercise intensity protocol was progressively overloaded, we compared the mean HR of the $W$ stage in every two protocol interval. The mean HR during protocols 7-12 was significantly higher than for protocols $1-6$ (Fig. 1B). Also, the mean resting HR was $83.8 \pm 6.3 \mathrm{bpm}$, and the highest HR recorded was $232 \mathrm{bpm}$ (data not shown).

Bone mineral density and muscle volume. BMD in the femur and vertebrae was measured using QCT before and after exercise. Post-exercise femoral BMD $(610.2 \pm 27.8 \mathrm{HA})$ increased significantly by $12.6 \%$ $(p<0.01)$ compared with pre-exercise BMD $(541.6 \pm 16.7 \mathrm{HA})$. In contrast, there was no difference in 
vertebral BMD, although exercise (317.2 $\pm 6.6 \mathrm{HA} ; 291.4 \pm 5.4 \mathrm{HA})$ increased BMD by $8.8 \%$. Also, MV values before and after exercise were not significantly different (Table 1 ).

Table 1

Analysis of bone mineral density and muscle volume before and after exercise in dogs. Bone mineral density and muscle volume data are represented as mean \pm SEM. ${ }^{*}$ Significant difference between pre-exercise and post-exercise $(p<$ $0.05)$. The rate of increase in parameters was calculated as (post-exercise mean - pre-exercise mean) $\div$ post-exercise mean $\times 100$.

\begin{tabular}{|llll|}
\hline Parameters (unit) & Pre-exercise & Post-exercise & $\begin{array}{l}\text { Rate of increase } \\
\text { (\%) }\end{array}$ \\
\hline Femur (HA) & $541.6 \pm 16.7$ & $610.2 \pm 27.8^{*}$ & 12.6 \\
\hline Vertebra (HA) & $291.4 \pm 5.4$ & $317.2 \pm 6.6$ & 8.8 \\
\hline Muscle volume $(\mathrm{HU})$ & $5384.0 \pm 890.7$ & $5434.0 \pm 740.3$ & 0.9 \\
\hline
\end{tabular}

Hematological and serum biochemistry parameters. Table 2 shows the differences in hematological and serum biochemistry parameters between pre-exercise and post-exercise. Levels of total alkaline phosphatase (TALP) as a serum bone marker showed a significant increase in post-exercise compared with pre-exercise $(p<0.01)$, but calcium and phosphorus levels were not different. Both AST and CK levels increased significantly after the exercise. All hematological and serum biochemistry parameters in preexercise and post-exercise dogs were within the reference range. 
Table 2

Analysis of serum biochemistry in dogs. Serum biochemistry data are represented as mean \pm SEM. *Significant difference between pre-and postexercise measures $(p<0.05)$.

\begin{tabular}{|llll|}
\hline Parameters (unit) & Preexercise & Post-exercise & Reference range \\
\hline Calcium (mg/L) & $9.5 \pm 0.2$ & $9.0 \pm 0.0$ & $9.0-11.9$ \\
\hline Phosphorus (mg/L) & $3.9 \pm 0.2$ & $4.0 \pm 0.2$ & $1.3-6.3$ \\
\hline TALP (U/L) & $68.6 \pm 9.2$ & $81.3 \pm 17.2^{*}$ & $0-97.9$ \\
\hline ALT (U/L) & $36.3 \pm 3.7$ & $41.0 \pm 5.0$ & $5.8-83.3$ \\
\hline AST (U/L) & $23.5 \pm 1.0$ & $33.5 \pm 1.6 *$ & $11.7-42.5$ \\
\hline BUN (mmol/L) & $14.3 \pm 9.3$ & $12.3 \pm 0.7$ & $9.6-31.4$ \\
\hline Creatinine (mg/L) & $0.7 \pm 0.0$ & $0.7 \pm 0.0$ & $0.4-1.3$ \\
\hline Glucose (mmol/L) & $94.8 \pm 3.2$ & $96.3 \pm 5.4$ & $74.5-120$ \\
\hline Albumin (g/dL) & $3.8 \pm 0.1$ & $3.8 \pm 0.0$ & $2.6-4.4$ \\
\hline Total protein (g/dL) & $6.8 \pm 0.2$ & $6.7 \pm 0.2$ & $5.7-7.5$ \\
\hline Cholesterol (mg/L) & $218.5 \pm 23.0$ & $208.0 \pm 25.3$ & $112-312$ \\
\hline Triglycerides (mmol/L) & $59.8 \pm 9.8$ & $63.6 \pm 12.8$ & $21-133$ \\
\hline CK (U/L) & $114.8 \pm 5.3$ & $214.0 \pm 20.8 *$ & $8-216$ \\
\hline
\end{tabular}

Correlations between bone mineral density, muscle volume, and serum biochemistry parameters. Figure 2 illustrates the relationship between TALP and BMD in the femur and vertebrae and MV in the thigh. We found a significant correlation between TALP and BMD in the femur $(r=0.760 ; p=0.004)$ and vertebrae $(r$ $=0.637 ; p=0.025)$. We also found a positive relationship between TALP and MV $(r=0.595 ; p=0.041)$. Those results provide evidence that exercise-induced increases in TALP are associated with increases in BMD and MV.

\section{Discussion}

The main objective of this study was to examine the effects of long-term interval exercise on HR, BMD, $\mathrm{MV}$, and serum biochemistry parameters in healthy dogs. A primary finding was that the HR response to interval treadmill exercise in different stages and protocols was normal and affirmative. A secondary finding of our study indicates that interval exercise enhances BMD in the femur and increases TALP, aspartate aminotransferase, and creatine kinase biomarkers. To the best of our knowledge, these are the first findings indicating that long-term interval exercise training is feasible for dogs and improves BMD in 
the femur. We suggest that increased TALP levels may be an associated mechanism of increasing BMD with exercise in dogs.

Ferasin et al. ${ }^{25}$ showed that dogs frequently refuse to exercise on the treadmill and are easily distracted in a laboratory environment. Due to those tendencies, adequate acclimatization is needed before initiating the exercise program. In this study, the beagles did not show any rejective or maladaptive behaviors. Also, the treadmill interval exercises did not cause any side effects or adverse reactions in healthy dogs. All dogs were able to complete the exercise program and were in good physical condition. Our results are consistent with our previous findings of normal physiological and behavioral responses to treadmill exercise ${ }^{26}$. This may be because the dogs had a sufficient adaptive period on the treadmill, a well-designed exercise program was used, and adequate animal care was provided by the study veterinarians and researchers. Under these stringent experimental conditions, we aimed to explore the potential effect of long-term interval exercise on $\mathrm{HR}, \mathrm{BMD}, \mathrm{MV}$, and serum biochemistry parameters in beagles.

The mean HRs of all dogs who performed interval exercise for 12 weeks had changed according to protocol intensity and progress. Also, following the FITT-VP principle, we were able to identify a regular HR change by organizing a suitable exercise program for dogs. Unlike other dog studies that found irregular HR patterns that were not proportional to the activity and external stimulus ${ }^{27,28}$, our results showed a normal HR response according to interval exercise, which may be because we created an optimal research environment by providing proper controls to anticipate the dogs' sensitivities to sounds and odors. The continuous and systematic HR change that we observed was within a maximum HR 230 $\mathrm{bpm}^{7}$ during the interval exercise; this validates that the exercise program would be effective for improving cardiovascular health for dogs.

Next, we explored BMD and MV to evaluate whether an adaptive HR response to the exercise training protocol was beneficial to bone and muscle health. Our findings corroborate previous evidence that interval exercise can increase BMD. The $12.6 \%$ increase in BMD that we observed is consistent with human studies that found an association between BMD and injury ${ }^{29}$ or disease ${ }^{30}$. In humans, BMD is a key measure for diagnosing osteoporosis ${ }^{30}$, and a $3-5 \%$ increase in BMD has been shown to reduce fracture risk by $20-30 \%{ }^{30}$. There are few studies of the effect of exercise on BMD in dogs and those studies found that aerobic exercise training in dogs resulted in either decreased or unchanged $\mathrm{BMD}^{31,32}$. In contrast, several human studies confirmed an increase in post-exercise BMD, regardless of age and $\operatorname{sex}^{18}$. The cause of this discrepancy is not clear, but the inclusion of intensity as a FITT component might be important because intensity induces a BMD increase and, thus, is a primary influence on the extent of training effect ${ }^{33}$. Currently, the optimal intensity level for interval exercise to enhance BMD in dogs is not known ${ }^{31}$, but in humans, the proper endurance exercise intensity has been estimated to 55$75 \%$ of $\mathrm{HR}_{\max }{ }^{34}$. In this study, the mean HR during the workout stage was $158.2 \mathrm{bpm}$, and the dogs continued to exercise for 36 minutes for twelve weeks. A previous study reported $230 \mathrm{bpm}$ for $\mathrm{HR}_{\max }$ in their study dogs ${ }^{7}$; thus, the intensity of interval exercise imposed on each dog in this study was 
approximately $68.7 \%$ of $\mathrm{HR}_{\max }$. Furthermore, the combination of FITT components with progressive and overloading workouts may be associated with the BMD improvements observed in our study dogs.

The benefits of regular exercise on BMD may be primarily linked to mechanical loading mechanisms ${ }^{35}$. Evidence for the Mechanostat Theory of mechanical loading has been confirmed in several animal studies $^{36,37}$. Rats are tetrapodal animals that are known to have higher tibia stress because the tibia is subjected to greater weight-bearing during treadmill exercise compared with the vertebrae ${ }^{38}$. In a previous study of rats, treadmill exercise increased tibial BMD but not vertebral BMD ${ }^{39}$. Dogs, like rats, are tetrapodal animals, and their femurs are more likely to receive mechanical loads and to be more weightbearing than the vertebrae when running on treadmills ${ }^{39}$. Our findings are consistent with the results of other animal studies and also support the concept that weight-bearing activity has a positive influence on bone health ${ }^{40}$.

Many studies have suggested that treadmill exercise improves BMD, but the precise underlying molecular mechanism remains elusive ${ }^{35}$. Here, we examined the effects of treadmill exercise on serum bone markers such as calcium, phosphorus, and TALP to identify biological mechanisms. We found that exercise-induced increases in TALP are associated with increases in BMD. TALP is a critical biomarker to assess BMD accurately and efficiently in the absence of liver disease ${ }^{41}$. Several isoenzymes of TALP exist in various organs besides bone (e.g., liver, kidney), and serum TALP, derived mostly from bones, reflects the sum of those isoenzymes ${ }^{42}$. Particularly in young dogs, changes in TALP result from a bonespecific isoenzyme ${ }^{43}$, because $96 \%$ of TALP consists of this bone-specific isoenzyme ${ }^{44}$. The bonespecific isoenzyme exists on the plasma membrane of osteoblasts and is carried through systemic circulation during the bone mineralization process ${ }^{45}$. TALP plays a role in the hydrolysis of inorganic pyrophosphate and then generates inorganic phosphate to maintain the appropriate ratio of inorganic pyrophosphate to inorganic phosphate, which is essential for the mineralization process ${ }^{46}$. Therefore, the upregulation of TALP in the two-year-old dogs from this study is considered a positive biomarker associated with increased BMD.

Also, correlation analysis revealed a positive association between TALP and MV in exercised dogs. To our knowledge, this is the first report to identify a significant correlation between TALP and MV in exercised dogs. However, the cause of these consequences is not known, but exercise-induced crosstalk between muscles and bones may be involved. Further research is warranted.

\section{Conclusions}

We demonstrate that interval exercise has a positive impact on BMD in healthy dogs, and exerciseinduced enhancement of BMD is associated with increased TALP levels. Also, this study confirmed that QCT could be used as a measure to assess subtle changes in MV and BMD after a machine-running exercise intervention. Further investigations are needed to determine the impact of exercise on cardiovascular fitness-, bone-, and muscle-related genes in dogs. Such research would improve our 
understanding of bone-exercise mechanisms and bone-muscle-interaction mechanisms, which would yield fundamental insights into key challenges in exercise science research.

\section{Materials And Methods}

Animals. Six healthy beagles were included in this study, and information on these dogs is provided in Table 3. All dogs were cared for following the recommendations described in The Guide for the Care and Use of Laboratory Animals. The study was approved by the Institutional Animal Care and Use Committee of Hanyang University and Seoul National University (2020-0073A, SNU-180731-2). All methods and protocols were carried out in accordance with the relavant guidelines and regulations. Before initiation of the experiments, baseline hematological analyses and body composition screening were undertaken by a veterinarian. Also, all beagles were subject to the same dietary and resting conditions. The dogs were housed in an environment with 12 hours (07:00-19:00) of bright light and 12 hours (19:00-07:00) of the dark. The temperature of the breeding room was $22^{\circ} \mathrm{C}-23^{\circ} \mathrm{C}$, with $50-60 \%$ humidity. The dogs were kept in separate cages $(775 \times 960 \times 900 \mathrm{~cm})$ with soft rubber flooring that was cleaned daily. Meals were served twice a day $(09: 00,17: 00)$, and freshwater was provided freely. The dogs were not provided with food for four hours prior to exercise testing to prevent exercise-induced gastrointestinal distress, heartburn, and acid reflux.

Table 3

Characteristics of study dogs. Age and weight data are represented as mean \pm SD.

\begin{tabular}{|ll|}
\hline Parameters (unit) & Dogs \\
\hline No. of Dogs & 6 \\
\hline Sex & Male \\
\hline Age (month) & $29.1 \pm 6.7$ \\
\hline Weight (kg) & $10.9 \pm 0.5$ \\
\hline
\end{tabular}

Treadmill adaptation for dog safety. All dogs underwent two weeks of adaptive training to get acquainted with the researcher, laboratory environment, and exercise regimen in advance. The exercise training equipment included a treadmill (EGOJIN XG-V6E, Gyeonggi-do, Korea) and a safety belt, which was applied to each dog's chest. Rectal temperature was taken from each dog with a digital thermometer before and after exercise. Throughout the experiment, the researcher and veterinarian screened the dogs' behavior and confirmed their safety.

Interval exercise program. As a warm-up, the dogs performed a walking exercise for five minutes at 2$3 \mathrm{~km} /$ hour prior to interval exercise. The exercise training program consisted of 12 treadmill protocols, which are detailed in Fig. 3. Each protocol was repeated twice per week for 12 weeks. The protocol 
consisted of a workout stage (W) and an incomplete resting stage (R). Exercise intensity was gradually increased by changing treadmill grade and speed.

Heart rate measurement. A Polar H10 HR measuring device and monitor (Polar Electro Oy, Kempele, Finland) were used to evaluate HR response during interval exercise. The dogs wore HR measuring devices on their chests, and HR data were collected every second. The mean HR value for each stage in all protocols was analyzed using the Polar Flow Software program (Polar Electro Oy, Kempele, Finland).

Quantitative computed tomography (bone mineral density and muscle volume). QCT was used to measure BMD and MV. All dogs were fasted for at least six hours prior to QCT scan. The dogs were intravenously premedicated with glycopyrrolate (Mobinul; Myungmoon Pharm., Seoul, Korea) at $0.01 \mathrm{mg} / \mathrm{kg}$ and then anesthetized with $6 \mathrm{mg} / \mathrm{kg}$ of propofol (Provive; Myungmoon Pharm., Seoul, Korea). They were kept sedated with $1.5 \%$ isoflurane (Foran solution; Choongwae Pharm., Seoul, Korea) and received $100 \%$ oxygen via endotracheal tube intubation. Percentage of oxygen saturation, end-tidal $\mathrm{CO}_{2}$, and HR were routinely monitored. QCT was performed using a 16-channel multidetecting CT scanner (Brivo 385; GE Medical System, Milwaukee, WI, USA). The lumbar vertebrae and femur were scanned with the dogs in dorsal recumbency. A calibrated QCT phantom (QRM-BDC/3; QRM GmbH, Moehrendorf, Germany) was placed under each dog. The scanning parameters were set as follows: $100 \mathrm{kV}, 100 \mathrm{mAs}$, and 1.25-mm slice thickness and interval. The phantom and lumbar vertebrae were positioned parallel to each other. All QCT images were scanned using the bone algorithm. CT scan was performed before and after exercise (Fig. 1). All CT images were analyzed using commercially available software (RadiAnt DICOM viewer; Medixant, Poznan, Poland; Osirix DICOM viewer; Pixmeo, Geneva, Switzerland). The region of interest for QCT included only the vertebral body in the 3rd lumbar vertebra and was measured using an image at the origin of the transverse process. The cortical and trabecular bone at all measurement sites were included in the ROI. Femoral BMD was measured in the middle of the femoral neck, including one-third of the proximal diaphysis and one-third of the distal diaphysis. BMD was calculated from the $\mathrm{CT}$ image in Hounsfield units. MV was measured at the correct position using multi planar reconstruction at the center of the femur, and a cross-section perpendicular to the bone was obtained. Each variable was measured three times, and the mean value for each was obtained.

Hematology and serum biochemistry parameter analysis. Blood samples for hematological and serum biochemical parameter analyses were collected the day before protocol one initiation and one day after protocol 12 completion (Fig. 4). Blood samples were kept in tubes coated with lithium heparin and stored at $4{ }^{\circ} \mathrm{C}$. After blood withdrawal and plasma harvest, heparinized blood samples were allowed to clot and were then centrifuged to obtain serum. All analyses were performed within the first six hours after blood extraction. Hematological parameters were measured from EDTA-blood samples using ADVIA 2120i (NYN Tarrytown, Tarrytown, NY, USA). Biochemistry parameters were measured from heparinized plasma using the Hitachi 7180 Auto analyzer (Hitachi, Tokyo, Japan) with reagents specifically designed for the instrument. 
Statistical analyses. All analyses were performed with GraphPad Prism 5.0 (GraphPad Inc., La Jolla, CA, USA). A one-way repeated analysis of variance was used to determine the mean difference in HR, followed by a Bonferroni post-hoc test. The mean difference in BMD, MV, and serum biochemistry parameters between pre-exercise and post-exercise was assessed using a two-tailed Student's t-test. To determine the relationship between TALP and BMD and MV, we performed Spearman's correlation and linear regression analyses. Values are expressed as means \pm SEMs, and a P-value $<0.05$ was considered statistically significant.

\section{Declarations}

\section{Acknowledgment}

This study was performed with the support of the Cooperative Research Program for Agriculture Science and Technology Development (a supportive managing project of the Center for Companion Animals Research, \#PJ013959022019, \#PJ014759022021) and the Rural Development Administration of the Republic of Korea. We would like to thank Prof. Jae Hwan Kim for his technical assistance.

\section{Author contributions}

J.-H.K., H.J.O., and H.S.L. conceptualized and designed the research; H.S.L. performed experiments; H.S.L. analyzed data; J.-H.K., H.J.O., and H.S.L. interpreted experimental results; J.-H.K. and H.S.L. wrote the first draft of the manuscript; J.-H.K., H.J.O., and H.S.L. edited and revised the manuscript. J.-H.K. and H.J.O. contributed equally as correspondence authors. All authors have read and agreed to submit the manuscript and declare that there is no conflict of interest. The results of the present study are presented ethically, without plagiarism, tampering, or manipulation by the researchers.

\section{Data availability}

All data used to support the findings of this study are included within the article. The analyzed data during the current study are available from the corresponding author upon reasonable request.

\section{References}

1 MacKinnon, M. 'Sick as a dog': zooarchaeological evidence for pet dog health and welfare in the Roman world. World Archaeo/42, 290-309, doi:Pii 92154911310.1080/00438241003673011 (2010).

2 Bartges, J., Kushner, R. F., Michel, K. E., Sallis, R. \& Day, M. J. One Health Solutions to Obesity in People and Their Pets. J Comp Patho/156, 326-333, doi:10.1016/j.jcpa.2017.03.008 (2017).

3 Mosier, J. E. Effect of Aging on Body Systems of the Dog. Vet Clin N Am-Smal/19, 1-12 (1989).

4 Lee, H. S., Oh, H. J., Lee, S. H., Kim, J. W. \& Kim, J.-H. J. K. J. o. S. S. Comparison of physiological and hematological responses to treadmill exercise in younger and older adult dogs. 30, 677-688 (2019). 
5 Piccione, G., Casella, S., Panzera, M., Giannetto, C. \& Fazio, F. Effect of Moderate Treadmill Exercise on Some Physiological Parameters in Untrained Beagle Dogs. Exp Anim Tokyo61, 511-515, doi:DOI 10.1538/expanim.61.511 (2012).

6 Rovira, S., Munoz, A., Riber, C. \& Benito, M. Heart rate, electrocardiographic parameters and arrhythmias during agility exercises in trained dogs. Rev Med Vet-Toulouse161, 307-313 (2010).

7 Radin, L. et al. Heart rate deflection point during incremental test in competitive agility border collies. Vet Res Commun39, 137-142, doi:10.1007/s11259-015-9634-5 (2015).

8 Rizzo, M. et al. Monitoring changes in body surface temperature associated with treadmill exercise in dogs by use of infrared methodology. J Therm Bio/69, 64-68, doi:10.1016/j.jtherbio.2017.06.007 (2017).

9 Restan, A. Z. et al. Lactate and glucose thresholds and heart rate deflection points for Beagles during intense exercise. Am J Vet Res80, 284-293, doi:DOI 10.2460/ajvr.80.3.284 (2019).

10 Bilezikian, J. P. Osteoporosis in men. J Clin Endocr Metab84, 3431-3434, doi:DOI 10.1210/jc.84.10.3431 (1999).

11 Kuwabara, A. et al. High prevalence of vitamin K and D deficiency and decreased BMD in inflammatory bowel disease. Osteoporosis Int20, 935-942, doi:10.1007/s00198-008-0764-2 (2009).

12 Valimaki, V. V., Loyttyniemi, E. \& Valimaki, M. J. Quantitative ultrasound variables of the heel in Finnish men aged 18-20 yr: predictors, relationship to bone mineral content, and changes during military service. Osteoporosis Int17, 1763-1771, doi:10.1007/s00198-006-0186-y (2006).

$13 \mathrm{Kida}, \mathrm{K}$. et al. The exercise training effects of skeletal muscle strength and muscle volume to improve functional capacity in patients with myocardial infarction. Int J Cardio/129, 180-186, doi:10.1016/j.ijcard.2008.04.031 (2008).

14 Laredo, J. D., el Quessar, A., Bossard, P. \& Vuillemin-Bodaghi, V. Vertebral tumors and pseudotumors. Radiol Clin North Am39, 137-163, doi:10.1016/s0033-8389(05)70267-0 (2001).

15 Lester, M. E. et al. Influence of exercise mode and osteogenic index on bone biomarker responses during short-term physical training. Bone45, 768-776, doi:10.1016/j.bone.2009.06.001 (2009).

16 Burgomaster, K. A. et al. Similar metabolic adaptations during exercise after low volume sprint interval and traditional endurance training in humans. J Physiol-London586, 151-160, doi:10.1113/jphysiol.2007.142109 (2008).

17 Farias, L. F. et al. Effect of Low-Volume High-Intensity Interval Exercise and Continuous Exercise on Delayed-Onset Muscle Soreness in Untrained Healthy Males. Journal of Strength and Conditioning Research33, 774-782, doi:10.1519/Jsc.0000000000002059 (2019). 
18 Alghadir, A. H., Aly, F. A. \& Gabr, S. A. Effect of moderate aerobic training on bone metabolism indices among adult humans. Pak J Med Sci30, 840-844 (2014).

19 Bell, K. E., Seguin, C., Parise, G., Baker, S. K. \& Phillips, S. M. Day-to-Day Changes in Muscle Protein Synthesis in Recovery From Resistance, Aerobic, and High-Intensity Interval Exercise in Older Men. $J$ Gerontol a-Bio/70, 1024-1029, doi:10.1093/gerona/glu313 (2015).

20 Rauch, F. et al. Analysis of cancellous bone turnover by multiple slice analysis at distal radius - A study using peripheral quantitative computed tomography. J Clin Densitom4, 257-262, doi:Doi 10.1385/Jcd:4:3:257 (2001).

21 Schoenau, E., Neu, C. M., Mokov, E., Wassmer, G. \& Manz, F. Influence of puberty on muscle area and cortical bone area of the forearm in boys and girls. J Clin Endocr Metab85, 1095-1098, doi:DOI 10.1210/jc.85.3.1095 (2000).

22 De Decker, S., Lam, R., Packer, R. M. A., Gielen, I. M. V. L. \& Volk, H. A. Thoracic and Lumbar Vertebral Bone Mineral Density Changes in a Natural Occurring Dog Model of Diffuse Idiopathic Skeletal Hyperostosis. Plos One10, doi:ARTN e012416610.1371/journal.pone.0124166 (2015).

23 Chalmers, H. J. et al. Assessment of bone mineral density of the femoral head in dogs with early osteoarthritis. Am J Vet Res67, 796-800, doi:DOI 10.2460/ajvr.67.5.796 (2006).

24 Mitsiopoulos, N. et al. Cadaver validation of skeletal muscle measurement by magnetic resonance imaging and computerized tomography. J Appl Physio/85, 115-122 (1998).

25 Ferasin, L. \& Marcora, S. A pilot study to assess the feasibility of a submaximal exercise test to measure individual response to cardiac medication in dogs with acquired heart failure. Vet Res Commun31, 725-737, doi:10.1007/s11259-007-3566-7 (2007).

26 Lee, H. S. et al. Development of Novel Continuous and Interval Exercise Programs by Applying the FITT-VP Principle in Dogs. ScientificWorldJourna/2020, 3029591, doi:10.1155/2020/3029591 (2020).

27 Miyazaki, H. et al. Ranges of diurnal variation and the pattern of body temperature, blood pressure and heart rate in laboratory beagle dogs. Exp Anim Tokyo51, 95-98, doi:DOI 10.1538/expanim.51.95 (2002).

28 Beerda, B., Schilder, M. B. H., van Hooff, J. A. R. A. M., de Vries, H. W. \& Mol, J. A. Behavioural, saliva cortisol and heart rate responses to different types of stimuli in dogs. Appl Anim Behav Sci58, 365-381, doi:Doi 10.1016/S0168-1591(97)00145-7 (1998).

29 Gomez-Bruton, A., Matute-Llorente, A., Gonzalez-Aguero, A., Casajus, J. A. \& Vicente-Rodriguez, G. Plyometric exercise and bone health in children and adolescents: a systematic review. World J Pediatr13, 112-121, doi:10.1007/s12519-016-0076-0 (2017). 
30 Berry, S. D. et al. Changes in bone mineral density (BMD): a longitudinal study of osteoporosis patients in the real-world setting. Arch Osteoporos13, doi:ARTN 12410.1007/s11657-018-0528-3 (2018).

31 Puustjarvi, K. et al. Endurance Training Associated with Slightly Lowered Serum Estradiol Levels Decreases Mineral Density of Canine Skeleton. J Bone Miner Res7, 619-624 (1992).

32 Hamrick, M. W., Skedros, J. G., Pennington, C. \& McNeil, P. L. Increased osteogenic response to exercise in metaphyseal versus diaphyseal cortical bone. J Musculoskelet Neuronal Interact6, 258-263 (2006).

33 Liang, M. T. C. et al. Effect of High-Impact Aerobics and Strength Training on BMD in Young Women Aged 20-35 Years. Int J Sports Med32, 100-108, doi:10.1055/s-0030-1268503 (2011).

34 Ghasemalipour, H., \& Eizadi, M. The Effect of Aerobic Training on Some Bone Formation Markers (Osteocalcin, Alkaline Phosphatase) in Asthma Treated with Inhaled Corticosteroids. J Res Med Sci20, (2018).

35 Berman, A. G., Hinton, M. J. \& Wallace, J. M. Treadmill running and targeted tibial loading differentially improve bone mass in mice. Bone Rep10, 100195, doi:10.1016/j.bonr.2019.100195 (2019).

36 Wolff, J. J. A. H. Das gesetz der transformation der knochen. 1, 1-152 (1892).

37 Sun, X. L. et al. The Effects of Combined Treatment with Naringin and Treadmill Exercise on Osteoporosis in Ovariectomized Rats. Sci Rep-Uk5, doi:ARTN 1300910.1038/srep13009 (2015).

38 Yeh, J. K., Aloia, J. F., Chen, M. M., Tierney, J. M. \& Sprintz, S. Influence of Exercise on Cancellous Bone of the Aged Female Rat. J Bone Miner Res8, 1117-1125 (1993).

39 Iwamoto, J., Takeda, T. \& Ichimura, S. Effects of exercise on bone mineral density in mature osteopenic rats. J Bone Miner Res13, 1308-1317, doi:DOI 10.1359/jbmr.1998.13.8.1308 (1998).

40 Bennell, K. L. et al. Bone mass and bone turnover in power athletes, endurance athletes, and controls: A 12-month longitudinal study. Bone20, 477-484, doi:Doi 10.1016/S8756-3282(97)00026-4 (1997).

41 Rudberg, A., Magnusson, P., Larsson, L. \& Joborn, H. Serum isoforms of bone alkaline phosphatase increase during physical exercise in women. Calcified Tissue Int66, 342-347, doi:DOI $10.1007 / \mathrm{s} 002230010071$ (2000).

42 Tsuritani, I. et al. Serum Bone-Type Alkaline-Phosphatase Activity in Women Living in a CadmiumPolluted Area. Toxicol Lett71, 209-216 (1994).

43 Stockham, S. L. \& Scott, M. A. Fundamentals of veterinary clinical pathology. (John Wiley \& Sons, 2013).

44 Syakalima, M., Takiguchi, M., Yasuda, J. \& Hashimoto, A. The age dependent levels of serum ALP isoenzymes and the diagnostic significance of corticosteroid-induced ALP during long-term 
glucocorticoid treatment. J Vet Med Sci59, 905-909, doi:DOI 10.1292/jvms.59.905 (1997).

45 Gala, J., Diaz-Curiel, M., de la Piedra, C. \& Calero, J. Short- and long-term effects of calcium and exercise on bone mineral density in ovariectomized rats. Brit J Nutr86, 521-527, doi:Doi 10.1079/Bjn2001428 (2001).

46 Terkeltaub, R. A. Inorganic pyrophosphate generation and disposition in pathophysiology. Am J Physiol-Cell Ph281, C1-C11 (2001).

\section{Figures}

(A)

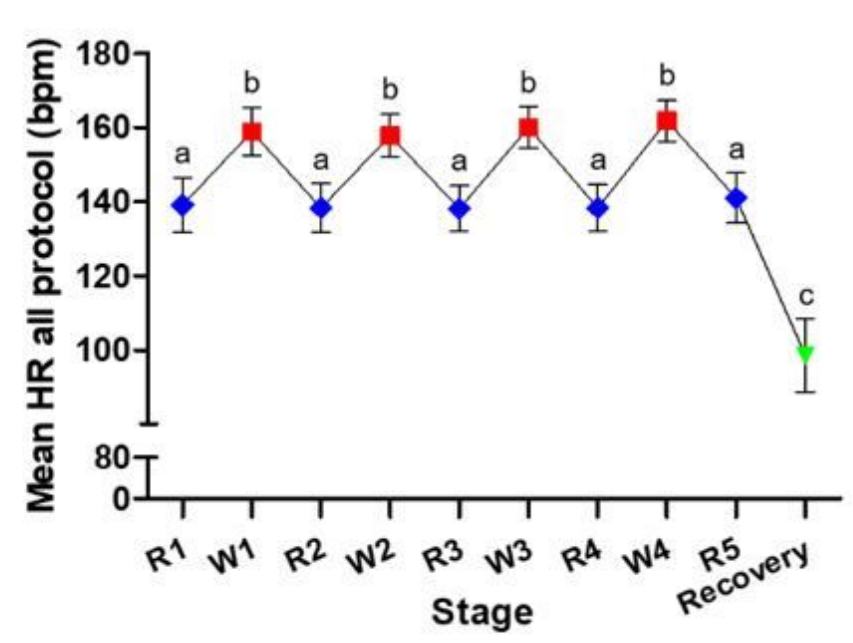

(B)

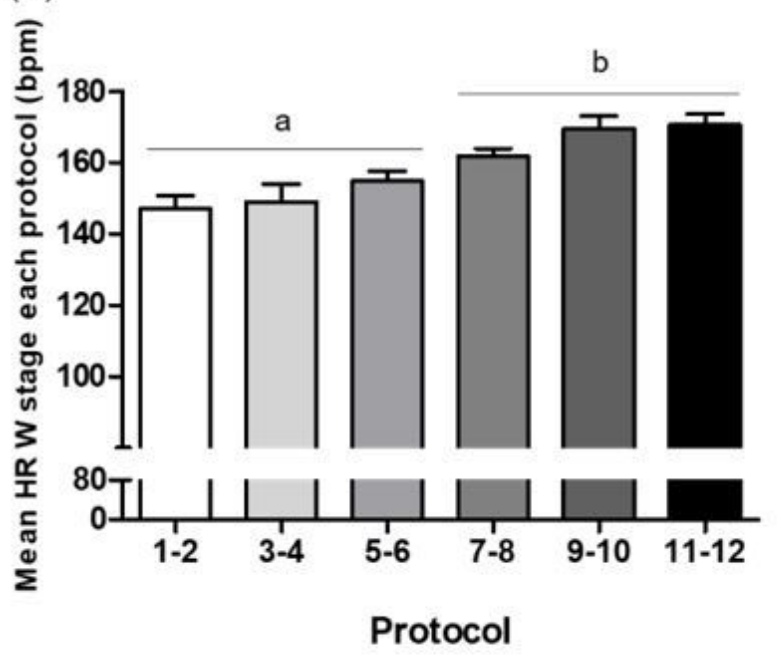

\section{Figure 1}

Analysis of mean heart rate during interval exercise training. (A) Mean heart-rate values for all dogs during incomplete resting stage (R1-R5), workout stage (W1-W4), and recovery. (B) Changes in mean heart rate during the $W$ stage according to treadmill exercise protocol (1-6 vs. 7-12). a, b, c Superscripts indicate significant differences when comparing the average heart rate of each stage or protocol $(p<$ 0.05). 
(A)

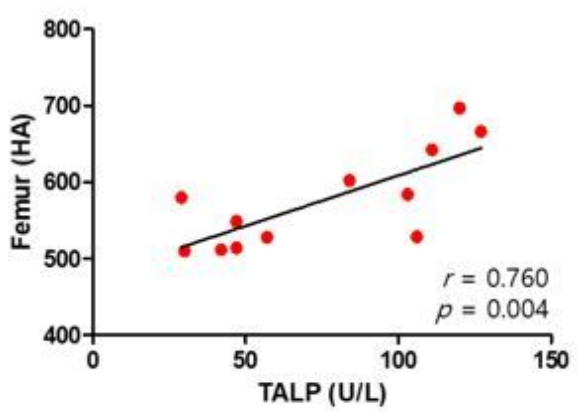

(B)

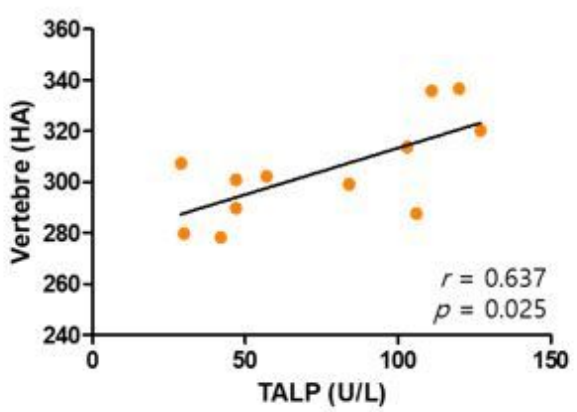

(C)

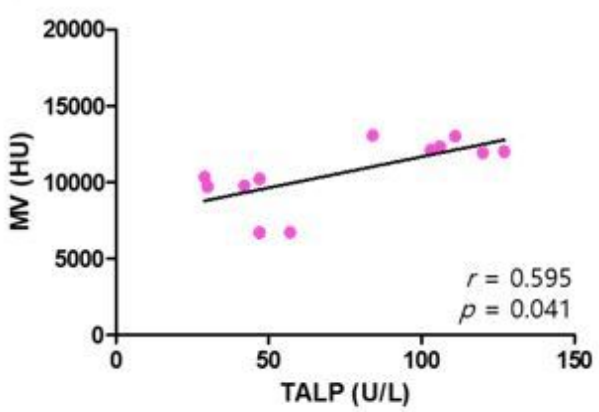

\section{Figure 2}

Correlations between total alkaline phosphatase (TALP) and bone mineral density (BMD) and muscle volume (MV) in dogs. (A) Correlation between TALP and individual BMD at the femur. (B) Correlation between TALP and individual BMD at the vertebrae. (C) Correlation between TALP and individual MV. 


\begin{tabular}{|c|c|c|c|c|c|c|c|c|c|c|c|}
\hline Protocol & Divide & $\mathbf{R} 1$ & W1 & $\mathbf{R} 2$ & w2 & $\mathbf{R 3}$ & w3 & R4 & W4 & R5 & Total time (min) \\
\hline \multirow{3}{*}{1} & Time (min) & 3 & 3 & 6 & 3 & 6 & 3 & 6 & 3 & 3 & \multirow{3}{*}{36} \\
\hline & Grade $(\%)$ & 0 & 2 & 0 & 2 & 0 & 2 & 0 & 2 & 0 & \\
\hline & Speed $(\mathrm{km} / \mathrm{h})$ & 4.8 & 7.4 & 4.8 & 7.6 & 4.8 & 7.8 & 4.8 & 8 & 4.8 & \\
\hline \multirow{3}{*}{2} & Time (min) & 3 & 3 & 6 & 3 & 6 & 3 & 6 & 3 & 3 & \multirow{3}{*}{36} \\
\hline & Grade (\%) & 0 & 2 & 0 & 2 & 0 & 4 & 0 & 4 & 0 & \\
\hline & Speed $(\mathrm{km} / \mathrm{h})$ & 4.8 & 7.4 & 4.8 & 7.6 & 4.8 & 7.8 & 4.8 & 8 & 4.8 & \\
\hline \multirow{3}{*}{3} & Time (min) & 3 & 3 & 6 & 3 & 6 & 3 & 6 & 3 & 3 & \multirow{3}{*}{36} \\
\hline & Grade (\%) & 0 & 4 & 0 & 4 & 0 & 4 & 0 & 4 & 0 & \\
\hline & Speed $(\mathrm{km} / \mathrm{h})$ & 4.8 & 7.6 & 4.8 & 7.8 & 4.8 & 8 & 4.8 & 8.2 & 4.8 & \\
\hline \multirow{3}{*}{4} & Time (min) & 3 & 3 & 6 & 3 & 6 & 3 & 6 & 3 & 3 & \multirow{3}{*}{36} \\
\hline & Grade $(\%)$ & 0 & 4 & 0 & 4 & 0 & 6 & 0 & 6 & 0 & \\
\hline & Speed $(\mathrm{km} / \mathrm{h})$ & 4.8 & 7.6 & 4.8 & 7.8 & 4.8 & 8 & 4.8 & 8.2 & 4.8 & \\
\hline \multirow{3}{*}{5} & Time (min) & 3 & 3 & 6 & 3 & 6 & 3 & 6 & 3 & 3 & \multirow{3}{*}{36} \\
\hline & Grade $(\%)$ & 0 & 6 & 0 & 6 & 0 & 6 & 0 & 6 & 0 & \\
\hline & Speed $(\mathrm{km} / \mathrm{h})$ & 4.8 & 7.8 & 4.8 & 8 & 4.8 & 8.2 & 4.8 & 8.4 & 4.8 & \\
\hline \multirow{3}{*}{6} & Time (min) & 3 & 3 & 6 & 3 & 6 & 3 & 6 & 3 & 3 & \multirow{3}{*}{36} \\
\hline & Grade (\%) & 0 & 6 & 0 & 6 & 0 & 8 & 0 & 8 & 0 & \\
\hline & Speed $(\mathrm{km} / \mathrm{h})$ & 4.8 & 7.8 & 4.8 & 8 & 4.8 & 8.2 & 4.8 & 8.4 & 4.8 & \\
\hline \multirow{3}{*}{7} & Time (min) & 3 & 3 & 6 & 3 & 6 & 3 & 6 & 3 & 3 & \multirow{3}{*}{36} \\
\hline & Grade (\%) & 0 & 10 & 0 & 10 & 0 & 10 & 0 & 10 & 0 & \\
\hline & Speed $(\mathrm{km} / \mathrm{h})$ & 4.8 & 9.2 & 4.8 & 9.6 & 4.8 & 10 & 4.8 & 10.4 & 4.8 & \\
\hline \multirow{3}{*}{8} & Time (min) & 3 & 3 & 6 & 3 & 6 & 3 & 6 & 3 & 3 & \multirow{3}{*}{36} \\
\hline & Grade $(\%)$ & 0 & 10 & 0 & 10 & 0 & 10 & 0 & 10 & 0 & \\
\hline & Speed $(\mathrm{km} / \mathrm{h})$ & 5 & 9.6 & 5 & 10 & 5 & 10.4 & 5 & 10.8 & 5 & \\
\hline \multirow{3}{*}{9} & Time (min) & 3 & 3 & 6 & 3 & 6 & 3 & 6 & 3 & 3 & \multirow{3}{*}{36} \\
\hline & Grade (\%) & 0 & 12 & 0 & 12 & 0 & 12 & 0 & 12 & 0 & \\
\hline & Speed $(\mathrm{km} / \mathrm{h})$ & 5.2 & 10 & 5.2 & 10.4 & 5.2 & 10.8 & 5.2 & 11.2 & 5.2 & \\
\hline \multirow{3}{*}{10} & Time (min) & 3 & 3 & 6 & 3 & 6 & 3 & 6 & 3 & 3 & \multirow{3}{*}{36} \\
\hline & Grade $(\%)$ & 0 & 12 & 0 & 12 & 0 & 12 & 0 & 12 & 0 & \\
\hline & Speed $(\mathrm{km} / \mathrm{h})$ & 5.4 & 10.4 & 5.4 & 10.8 & 5.4 & 11.2 & 5.4 & 11.6 & 5.4 & \\
\hline \multirow{3}{*}{11} & Time (min) & 3 & 3 & 6 & 3 & 6 & 3 & 6 & 3 & 3 & \multirow{3}{*}{36} \\
\hline & Grade (\%) & 0 & 14 & 0 & 14 & 0 & 14 & 0 & 14 & 0 & \\
\hline & Speed $(\mathrm{km} / \mathrm{h})$ & 5.6 & 10.8 & 5.6 & 11.2 & 5.6 & 11.6 & 5.6 & 12 & 5.6 & \\
\hline \multirow{3}{*}{12} & Time (min) & 3 & 3 & 6 & 3 & 6 & 3 & 6 & 3 & 3 & \multirow{3}{*}{36} \\
\hline & Grade $(\%)$ & 0 & 14 & 0 & 14 & 0 & 14 & 0 & 14 & 0 & \\
\hline & Speed $(\mathrm{km} / \mathrm{h})$ & 5.8 & 10.8 & 5.8 & 11.2 & 5.8 & 11.6 & 5.8 & 12 & 5.8 & \\
\hline
\end{tabular}

\section{Figure 3}

A 12-week interval exercise program consisting of 12 protocols. The 12 protocols include a gradual increase in grade $(\%)$ and speed $(\mathrm{km} / \mathrm{h})$ as the sessions proceed. 


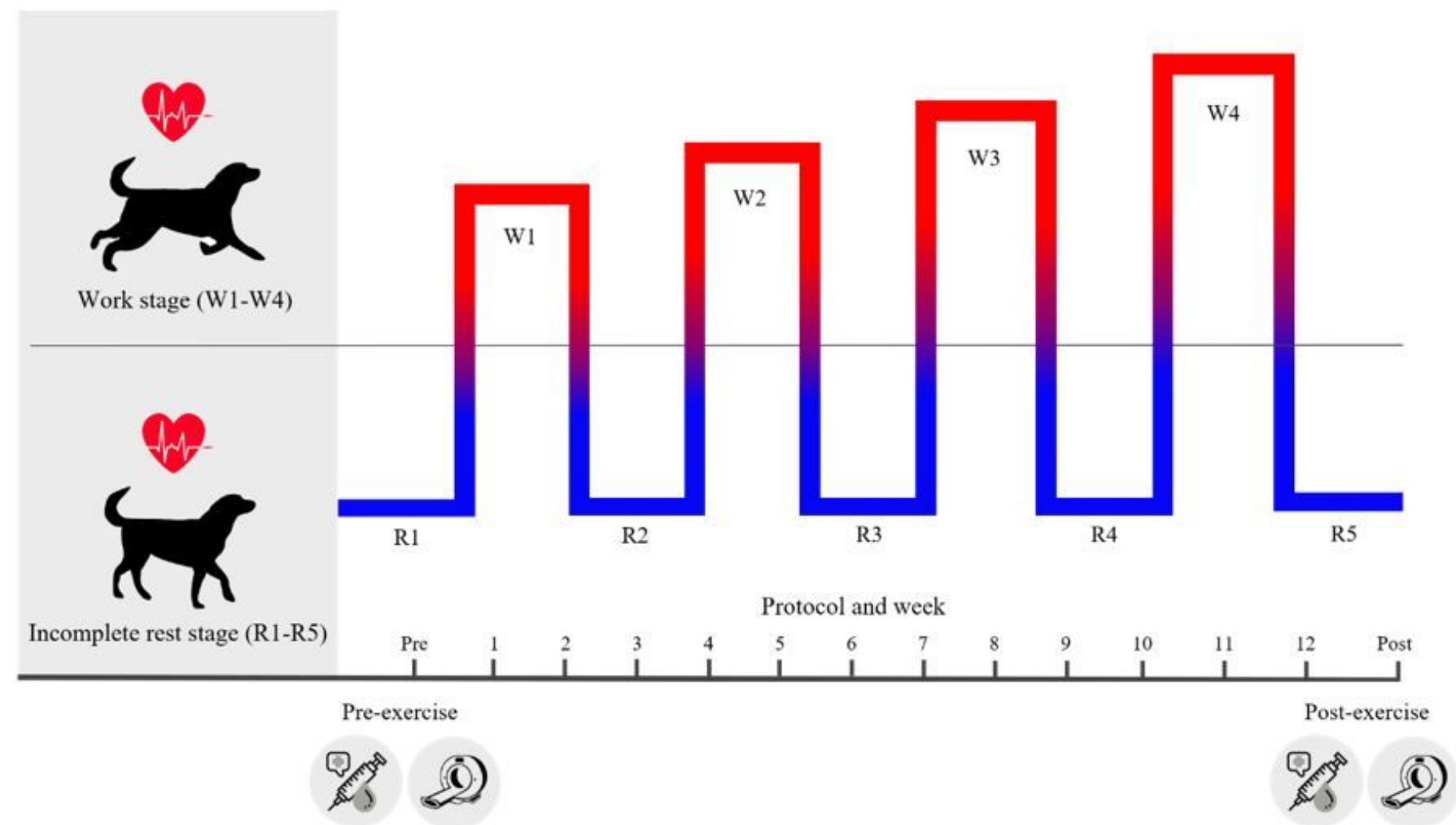

Figure 4

Schematic design of experimental procedures. Dogs underwent interval exercise comprising five resting stages and four workout stages over 12 weeks. Blood biochemical parameters (pre-exercise, postexercise), heart rate (overall interval exercise), and QCT (pre-exercise, post-exercise) were measured for each dog. 